\title{
Divas en la brega: apuntes para el estudio de Las divas de mi barrio de Carmen Montañez*
}

Fecha de recepción: 14 de septiembre de 2015

Fecha de aprobación: 15 de abril de 2015

\section{Resumen}

En este trabajo analizaré la manera en que Montañez redefine el concepto de diva en su colección de cuentos Las divas de mi barrio. Arguyo que su redefinición valoriza la función de personajes femeninos que no sólo difieren físicamente de las ideas de belleza que se asocian con una diva sino que pertenecen a la clase trabajadora del país donde se desarrolla la acción. Por lo tanto, en estos cuentos se rinde tributo a otras formas de femineidad y de conocimientos que tienden a quedar excluidos por no ir a tono con las conductas y estéticas que afianzan el machismo.

Palabras clave: Carmen Montañez, literatura puertorriqueña, diva, clase trabajadora, Arcadio Díaz Quiñones.

Citar: Lorenzo-Feliciano, V. (enero-junio de 2016). Divas en la brega: apuntes para el estudio de Las divas de mi barrio de Carmen Montañez. La Palabra, (28), 17-26. doi: http://dx.doi.org/10.19053/01218530.4785 


\section{Struggling divas: notes for a study of Las divas de mi barrio [Divas of my Neighborhood] by Carmen Montañez}

\section{Abstract}

In this paper I will discuss how Montañez redefines the term "diva" in her collection of short stories Las divas de mi barrio [Divas of my neighborhood]. I argue that this redefinition, values the role of female characters who differ physically from the ideas of beauty that tend to be associated with a diva. This is significant since these characters belong to the working class of the country where the plot develops. Thus, these short stories pay tribute to other forms of femininity and knowledge that tend to be excluded from the status quo for not going along with aesthetic preferences and behaviors that reinforce machismo.

Key words: Carmen Montañez, Puerto Rican literature, diva, working class, Arcadio Díaz Quiñones.

\section{Vedette en lutte: notes pour étude du livre Les vedettes de mon quartier de Carmen Montañez}

\section{Résumé}

Dans ce travail j'analyserai la manière comme Montañez redéfinit le concept de vedette dans son recueil de nouvelles Les vedettes de mon quartier. Je propose que sa redéfinition met en valeur la fonction des personnages féminins qui non seulement se différencient des idées de beauté physique qui s'associent à une vedette, mais aussi qu'appartiennent à une classe ouvrière du pays où se passe l'action. Par conséquence, dans ces nouvelles un hommage est rendu à d'autres formes de féminités et de connaissances qui souvent sont exclues parce qu'elles n'affirment pas des conduites et esthétiques du machisme.

Mots clés: Carmen Montañez, littérature portoricaine, vedette, classe ouvrière, Arcadio Díaz Quiñones. 


\section{Introducción}

A finales del siglo XIX, el término "diva" fue acuñado en Europa para referirse a las cantantes de ópera. Sin embargo, dicho término ha tenido una buena acogida en contextos sociales muy distintos a los del europeísmo decimonónico debido a que, como afirman Susan Leonardi y Rebecca Pope (1996) en su libro The Diva's Mouth, "no sólo cada estrella, cantante y artista ha sido llamada 'diva'. Las altas ejecutivas, las mujeres de bajo rango y las dragqueens también [son consideradas divas]" (pp. 1-2). ${ }^{1}$ La aseveración de Leonardi y de Pope enfatiza en que los términos evolucionan y con el tiempo adquieren distintos significados, razón por la cual en la actualidad "diva" no necesariamente alude a las cantantes de ópera.

Las divas -independientemente de si se encuentran en un contexto operístico o no- suelen ser el centro de atención en los escenarios, en los medios de comunicación, en las pasarelas, en carnavales, en escándalos y en círculos intelectuales. ${ }^{2}$ En algunos casos, esa ubicuidad en el centro ha permitido apoyar visiones alternativas a la opresión de las mujeres pero en otros, este enfoque ha implicado cierta complicidad con el sistema patriarcal que las oprime. En este aspecto, la crítica está dividida: hay quienes arguyen que una diva no tiene el potencial para llevar a cabo prácticas que resulten liberadoras para las mujeres; mientras que otros críticos sí plantean la posibilidad de que una diva presente un tipo de empoderamiento que socave estructuras sociales, culturales y políticas de índole represiva. Catherine Clément (1988) es de los académicos que afirman que una perspectiva liberadora de una diva es imposible: "La ópera concierne a las mujeres y no hay liberación. Todo lo contrario. Las mujeres sufren, lloran y mueren. Muy pocas divas, por no decir ninguna, escapan con vida"(p. 11). ${ }^{3}$ El argumento se desprende de su enfoque exclusivo al mundo operístico. Mucho más ricas resultan las propuestas ya mencionadas de Leonardi y de Pope, así como las de Smitherman y Brown, porque no se limitan al ámbito de la ópera y se enfocan en cómo una diva tiene cierta movilidad en la esfera pública.

Ahora bien, si el término "diva" tiene su origen en lo musical, ¿cómo se relaciona el mismo con lo literario? Cabe recalcar que su uso inicial en el plano literario sí se centraba en la prima donna. Una vez más, el libro de Leonardi y Pope es revelador en este caso puesto que provee un panorama que incluye, entre otros asuntos, investigaciones sobre las divas en novelas europeas y estado-unidenses. $\mathrm{Su}$ análisis de dicho corpus literario apunta a que hay una vertiente en la cual la cantante es presentada de modo negativo al ésta ser frívola, "devoradora de hombres", melodramática, vengativa y egoísta (Leonardi y Pope, 1996, pp. 47-49). Asimismo, hay otra variante de las novelas de prima donnas en las que éstas se presentan como seres talentosos pero débiles que necesitan tener la protección de un hombre. Sin embargo, hay una corriente positiva donde la prima donna está en control de su vida profesional, emocional y sexual sin depender de un hombre y sin dar rienda suelta a la venganza, al egoísmo y al melodrama. En otras palabras, esta diva se caracteriza por trabajar arduamente para desarrollar su talento y obtener fama por sus propios méritos (Leonardi y Pope, 1996, pp. 88, 97).

«not only has every female star, singer, and artist been dubbed diva... High-ranking business women...some pretty low-ranking women and...drag queens [are considered divas as well]». [La traducción al español es mía].

2 Emily Hind (2010) ha demostrado en su estudio sobre las escritoras mexicanas Guadalupe Amor, Elena Garro y Antonieta Rivas Mercado la conexión entre las divas y los círculos intelectuales. Hind usa el término «diva-lectuals» (i.e. «diva» + «intelectual») para referirse a estas escritoras. Ver su libro Femmenism and the Mexican Woman Intellectual from Sor Juana to Poniatowska: Boob Lit para más información sobre el tema.

3 «Opera concerns women...No, there is no liberation. Quite the contrary: they suffer, they cry, they die. None of them escape with their life, or very few of them do». [La traducción al español es mía]. 
En las letras hispánicas hay ejemplos importantes de cantantes de ópera como El final de Norma de Pedro Antonio de Alarcón (1855), Sin rumbo de Eugenio Cambaceres (1855), Su único hijo de Leopoldo Alás "Clarín" (1891) y Boca de lobo de Martha Bátiz (2007). ${ }^{4}$ Con todo, esta investigación tiene que ver con el uso del término "diva" para referirse a personajes que no se circunscriben al contexto musical, dado que este trabajo se centra en algunos cuentos de Las divas de mi barrio (2012) de la puertorriqueña Carmen Montañez. En los cuentos de Montañez se rinde tributo a otras formas de femineidad y de conocimientos que tienden a quedar excluidos por no ir a tono con conductas pasivas ante la violencia doméstica, ni con estéticas afianzadoras del machismo y el sistema consumista que promueve el gasto económico para tener un cuerpo "perfecto" para ser consumido visual y sexualmente por los hombres.

El uso que Montañez le otorga al término "diva" es significativo si consideramos que estudios como el de Leonardi y Pope demuestran que su uso indiscriminado no tiene la misma connotación en todos los casos, ya que suele usarse de manera positiva para referirse a unas mujeres pero puede tener connotaciones negativas para referirse a otras. ${ }^{5}$ Por lo tanto, arguyo que Montañez usa el término "diva" de modo no tradicional y positivo, puesto que valoriza la función de personajes femeninos de la clase trabajadora que difieren físicamente de los ideales artísticos y estéticos que se asocian con una diva.

Con esto en mente, enmarcaré Las divas de mi barrio en el contexto de la literatura puertorriqueña para subrayar que esta colección de cuentos rompe y continúa con ciertas características temáticas y estilísticas de las letras boricuas. Luego, examinaré algunos cuentos partiendo de lo que Arcadio Díaz Quiñones ha denominado "el arte de bregar", porque este acercamiento me permitirá estudiar los cuentos en el contexto sociocultural y político del momento.

\section{Contexto literario}

En Las divas de mi barrio hay rupturas y continuaciones con otros textos puertorriqueños, particularmente en lo que concierne al tropo de la enfermedad, la formación de subjetividades femeninas y la descripción de personajes de la clase obrera. Por ejemplo, en $L a$ guaracha del Macho Camacho Luis Rafael Sánchez (1976) contrasta a los personajes China Hereje y Doña Chon con Graciela Alcántara y López de Montefrío. La voz narrativa en la novela se identifica particularmente con Doña Chon -el personaje de la clase trabajadora- en vez de identificarse con Graciela, el personaje de clase alta con aires de diva. Sin embargo, podemos ir aún más atrás. Un recorrido por la cuentística puertorriqueña demuestra que ya desde los años cuarenta, escritores del cuño de José Luis González y Abelardo Díaz Alfaro se habían enfocado en el desarrollo de personajes proletarios. A finales de los sesenta se sigue elaborando esta temática pero con nuevos puntos de vista que difieren de los modelos establecidos por autores previos. Me refiero a que en cuentos más recientes cuyos personajes pertenecen a la clase trabajadora no se presenta a los mismos como víctimas, toda vez que se empieza a expresar de manera más abierta temas relacionados a la femineidad, al feminismo y a los papeles de género (Barradas, 1983, p. xxvii). Montañez continúa el hilo temático de cuentistas que publicaron antes que ella si se considera que en Las divas de mi barrio, aunque no se exalta el habla popular de la clase trabajadora, los personajes femeninos lidian o "bregan"

$4 \quad$ Aclaro que la prima donna en la novela Sin rumbo no es el personaje principal, pero tiene una función importante en el desarrollo de la trama.

5 Leonardi y Pope se refieren al contexto racial estadounidense en el cual para la mujer blanca ser una «diva» se ve de manera positiva, pero para la mujer negra ser una «diva» puede tener connotaciones de «parejera», «agresiva», «afrentá», «bitchy», etc. 
con distintos problemas sin ser víctimas. ${ }^{6}$

Ahora bien, la ruptura que percibo en la colección de cuentos de Montañez tiene que ver con el tropo de la enfermedad o discapacidad tan arraigado en la literatura puertorriqueña. Desde el siglo XIX, varios escritores incluyeron en sus respectivos discursos metáforas sobre enfermedades para aludir a la Isla. Este discurso de raíz decimonónica se encuentra en varios contextos latinoamericanos como, por ejemplo, los ensayos del boliviano Alcides Arguedas y del venezolano César Zumeta, a causa de que

La campaña para aislar y clasificar orgánicamente y socialmente al enfermo era parte de un proyecto mayor de racionalizar, modernizar e industrializar la nación. La función del discurso de la degeneración determinó cuáles grupos y prácticas constituían obstáculos biológicos y culturales para la modernidad, con el fin de diagnosticar las enfermedades que afectaban a estos grupos y desarrollar tratamientos o soluciones. (Aronna, 1999, p. 14)

En el caso de Puerto Rico, el apego a estas ideas ha sido recurrente. Si bien se tiende a pensar principalmente en las novelas naturalistas de Manuel Zeno Gandía que componen las Crónicas de un mundo enfermo, ya en 1880 Salvador Brau había incorporado propuestas de índole biológica en sus publicaciones. ${ }^{8}$ Su ensayo "Las clases jornaleras" contiene referencias genéticas y anatómicas con el fin de "[llevar] a cabo una disección del cuerpo social, reconocer sus males" y sugerir "una serie de prescripciones que delatan una intención moralizante, de reconstrucción social" (Álvarez Curbelo, 2002, p. 293). ${ }^{9}$ En el siglo XX, el tropo en cuestión resurge en textos canónicos como Insularismo de Antonio S. Pedreira (1934) y La guaracha del Macho Camacho de Luis Rafael Sánchez, debido a que la enfermedad se convierte en metáfora del colonialismo norteamericano (Gelpí, 2005, pp. 17,19$).^{10}$

En seis de los diez cuentos que componen Las divas de mi barrio hay casos de enfermedad, discapacidad o "anormalidad" corporal. No obstante, a diferencia de cómo se ha desarrollado este tropo en otros textos canónicos, en los de Montañez las enfermedades o discapacidades no están vinculadas a venganzas ni se convierten en impedimentos que limitan totalmente a los personajes femeninos que trabajan y "bregan" con sus problemas de salud. Es por eso que, en mi lectura de Las divas de mi barrio, estas condiciones médicas no necesariamente implican el estancamiento colonial que en varios textos puertorriqueños ha sido representado mediante

$6 \quad$ Para más información sobre el habla popular de la clase trabajadora en la cuentística puertorriqueña, ver el prólogo de Efraín Barradas en la antología Apalabramiento.

7 «the drive to isolate and classify the organically and socially ill was part of a greater project to rationalize, modernize, and industrialize the nation. Fulfilling this need, the function of the discourse of degeneration was to determine which groups and practices constituted biological and cultural obstacles to modernity, to diagnose the illnesses afflicting these groups and to develop treatments or solutions». La traducción al español es mía. Ver el libro de Michael Aronna «Pueblos enfermos»: The Discourse of IIIness in the Turn-of the-Century Spanish and Latin American Essay para más información sobre este enfoque biologicista en la ensayística iberoamericana de finales del siglo XIX.

8 Las cuatro novelas que componen Las crónicas de un mundo enfermo son La charca (1894), Garduña (1896), El negocio (1922) y Redentores (1925).

9 Para un estudio sobre la función de Salvador Brau en los proyectos decimonónicos de índole política y social en Puerto Rico, ver el último capítulo de Un país del porvenir: el afán de modernidad en Puerto Rico (siglo XIX) de Silvia Álvarez Curbelo. Para un excelente análisis de la metáfora del cuerpo enfermo en la literatura canónica puertorriqueña, ver Literatura y paternalismo en Puerto Rico de Juan Gelpí, donde se menciona a intelectuales como Eugenio María de Hostos que en algún momento incorporaron dicha metáfora en sus escritos.

10 Otro ejemplo vendría siendo «La muñeca menor» de Rosario Ferré donde se combinan la enfermedad, los cambios de clase social que surgieron a raíz de la invasión estadounidense de 1898, la venganza y lo fantástico. 
el tropo de la enfermedad o discapacidad. No obstante, para entender cómo en los cuentos de Montañez no se victimiza a los personajes pobres, enfermos y discapacitados, es necesario indagar en lo que implica el "arte de bregar".

\section{El arte de bregar}

Díaz Quiñones (2000) explica el uso puertorriqueño de "bregar" como la aceptación de los límites, la búsqueda de un acuerdo y la negociación dentro de un marco reducido de posibilidades (pp. 22-24). Esta aceptación, búsqueda y negociación sucede sin recurrir a ataques directos y sin perder la dignidad. Díaz Quiñones propone además tres ejes para analizar la brega: el trabajo concreto, el erótico y la negociación social o espiritual que conlleva actuar con sutileza (p. 28). Es curioso, pues, que el inicio de la brega puede situarse en "viejas prácticas de ocultamiento pertenecientes a épocas remotas de la sociedad cimarrona, a un mundo rural que se desarrolló en los márgenes del estado colonial, una sociedad de grandes penurias y bruscos desplazamientos" (Díaz Quiñones, 2000, p. 27). La referencia al cimarronaje es pertinente porque los personajes de Montañez se desarrollan en lo que hoy se conoce como Santurce, lugar que en la época colonial española se caracterizó, en parte, por los grupos de cimarrones que se establecieron en dicho espacio llamado, en aquel entonces, San Mateo de Cangrejos. Por otro lado, el hecho de que la práctica de bregar se origine al margen del estado es significativo si se toma en cuenta que las divas de Montañez, las cuales algunos podrían considerar estrafalarias, son personajes femeninos del margen con saberes y prácticas que les permiten manejar el arte de bregar para poder subsistir en ese margen.

Sin más, me concentraré en algunos casos en los cuales los personajes femeninos de la clase trabajadora ocupan el "center stage" y bregan dignamente con la pobreza, la enfermedad/ discapacidad, la violencia y otros problemas no asociados con la idea general que se tiene de una diva.

Bregando con la pobreza o la discapacidad

Los cuentos "Doña Virginia la curandera", "Doña María la que plancha" y "Doña Maximina la businesswoman" se enfocan en la discapacidad y en la parte de la brega que tiene que ver con el trabajo, particularmente el de índole manual. ${ }^{11}$ Por ejemplo, doña Maxi es la mejor alambiquera del barrio y diríase de todo el Caribe, razón por la que personas de distintas clases sociales acuden a su casa para buscar su famosa bebida alcohólica. En varias ocasiones, la policía allana su residencia y Maxi es arrestada por la fabricación de ron casero. Maxi, esta diva de quien se dice que "nadie recuerda haberla visto joven", no solamente subsiste mediante su trabajo sino que brega con el sistema legal que insiste en criminalizar su modo de ganarse la vida. Asimismo, Maxi brega con los que intentan arrebatarle el secreto de su arte para mercadear el producto sin su consentimiento (Montañez, 2012, p. 25). Su forma de preparar un producto clandestino y escapar de las autoridades remite a la fuga y al cimarronaje en el cual se sitúan los inicios de la brega en comunidades marginales (Díaz Quiñones, 2000, p. 54). Uno de los sucesos más significativos del cuento es cuando Maxi logra bregar con los policías y con el juez: "En ese momento aprovechaba doña Maxi para dejar la botella encima de la mesa del magistrado y salía como alma que lleva el diablo, con sus piernas una mirando para el norte y otra mirando para el sur" (Montañez, 2012, p. 29). Al parecer, el pleito criminal contra la businesswoman queda archivado cuando el magistrado prueba el exquisito ron. En resumen, en este cuento no

11 Por cuestiones de espacio, no discutiré en este análisis el cuento "Doña Virginia la curandera", pero lo menciono porque en el mismo la diva se gana el sustento con sus manos, las cuales cambian de tamaño a lo largo del relato. 
solamente la diva brega con la pobreza y con el sistema jurídico de su entorno, sino que de por sí desafía las nociones de belleza o femineidad que se asocian con una diva, puesto que este personaje femenino es zambo.

En "Doña María la que plancha", el arte de bregar no solamente se circunscribe a lo laboral sino que también entra el plano erótico (Díaz Quiñones, 2000, p. 24), toda vez que este personaje es de los pocos que se describen como portadores de una inusitada belleza física. María establece una relación con un hombre mayor y casado. Aunque el hombre sí contribuye con la manutención de los hijos que tiene con María, él nunca cumple su promesa de divorciarse para casarse con ella. El hombre fallece de repente; la herencia que recibe María apenas le alcanza para dos meses. A María -que bregó en el plano erótico con su amante-le toca bregar sola para levantar a sus cuatro hijos. Aquí, la brega sigue en el plano laboral, ya que María establece su propio negocio de planchadora y es tanta la fama que adquiere que logra ganar lo suficiente para subsistir. El hecho de que este personaje termine planchando para la tienda González Padín parece insignificante, pero es importante, porque dicho establecimiento es uno donde compraban mujeres de clase alta. Es decir, un personaje como María, que se encarga de que la ropa que se vende en González
Padín esté libre de arrugas y bien almidonada, no tiene el poder adquisitivo para comprar en ese lugar. Sin embargo, en este cuento, el enfoque no recae en las divas que sí pueden comprar ahí sino en alguien que trabaja arduamente para ese lugar con el fin de mantenerse. Por ende, el papel de diva se invierte en este caso y se trae al centro a un personaje que solamente posee su capacidad de trabajo.

A lo largo del relato, María continúa en la brega como madre soltera de la clase trabajadora pero le toca entonces enfrentarse a otro desafío: la discapacidad. A causa de un accidente, la pierna de María queda con una herida que no cicatriza. Esto dificulta muchísimo su trabajo hasta que un día uno de sus hijos milagrosamente la sana. No obstante, la brega en el plano de la salud continúa porque días más tarde su hijo tiene la herida en su pierna y ahora él debe lidiar con la discapacidad. Esto implica que "la vida una vez más le daba otra bofetada en la cara" a María (Montañez, 2012, p. 74).

Aunque el cuento concluye con esa expresión, hay que recordar que no es la primera vez que la vida le daba una bofetada a este personaje que previamente había triunfado ante la adversidad (nótese el uso de la expresión "una vez más" en la cita anterior). A lo que me refiero es a que más que a cierto estancamiento, la bofetada apunta al dolor de madre por tener un hijo con problemas de salud. Con todo, estos problemas no son totalmente discapacitantes a diferencia de, por ejemplo, la condición médica del hijo que la China Hereje tiene con su amante en La guaracha del Macho Camacho. Asimismo, si el hijo de María pudo sanar a su madre de modo sobrenatural, es posible que sus poderes curativos regresen y entonces él quede libre de dicha discapacidad.

El personaje Carmelina, la diva del cuento "Plegaria al santo patrón", también está en la brega ante la pobreza. En este cuento, uno de los pocos narrados en primera persona, se presenta a un personaje femenino que le reza a distintos santos y santas con tal de que le concedan el premio de la lotería. Aquí, el humor juega un papel importante dado que esta diva le reza a divas del establishment como Lady Di. La brega aquí estriba en negociar, en este caso con los santos, para sobrevivir. Esta faceta de "la brega" va a tono con lo que menciona Díaz Quiñones sobre el alivio que da el saber que hay un intermediario que va a "bregar" con las dificultades de uno (p. 21). En este contexto, la fe con que Carmelina reza brega a su favor y la plegaria se convierte en un acto de negociación con los santos (i.e. los intermediarios). La misma surte efecto y lleva a que Carmelina se gane la lotería con el número 666. 
Bregando con el abuso y la violencia de género

Ahora bien, no todos los cuentos tienen el humor de "Plegaria al santo patrón". Otro de los temas en esta colección de cuentos es la brega de algunas divas con el abuso y con la violencia de género. Es menester poner esto en contexto: Puerto Rico es un país donde las mujeres forman la mayoría de la población y casi todos los hogares con un solo guardián son regidos por ellas (Iriarte, 2015). Cada año se cometen atrocidades contra las mujeres que, muchas veces, la policía pasa por alto por considerarlas "cosas de pareja". Concuerdo con Mariana Iriarte (2015) cuando alude al vínculo entre la pobreza y la violencia que afecta a tantas mujeres, al explicar que: "La violencia afecta la capacidad productiva y de generar ingresos de las mujeres sumiéndolas en la pobreza. A su vez, la pobreza, en muchas ocasiones, las confina en relaciones abusivas, puesto que pueden no ver o encontrar maneras alternativas de mantener a sus hijos, hijas y a ellas mismas". En suma, en varios casos la pobreza y la violencia doméstica forman un fuerte vínculo que afecta negativamente la vida de muchas mujeres. No pretendo insinuar que el problema de la violencia de género es algo que solamente les ocurre a las personas de escasos recursos, dado que este mal afecta a individuos en todos los estratos sociales. A lo que me refiero es a que, como explica Iriarte, la violencia en un contexto de pobreza es más difícil de combatir debido a que ata económicamente a las mujeres a su agresor.

Si bien en Las divas de mi barrio hemos visto tres ejemplos de personajes femeninos que bregan con la pobreza o con la discapacidad, hay cuentos a los que se les añade la brega con la violencia. Por ejemplo, María de los Ángeles Mena es otra diva de la colección de cuentos que nos ocupa, quien ha tenido que bregar con la discapacidad y la violencia. En primer lugar, su madre nunca la quiso, dado que en el texto se insinúa que Mena fue producto de una violación. Es Mena quien paga los platos rotos por lo sucedido, pues un día su madre le derrama agua hirviendo encima y la desfigura. No obstante, este personaje pobre logra educarse y convertirse en maestra gracias a una vecina que se hizo cargo de ella tras la muerte de su madre.

Mena brega satisfactoriamente con las marcas de la quemadura, con la fealdad que la caracteriza y con las miradas de los curiosos: "Con la madurez que da el tiempo, superé las miradas curiosas [...] La fealdad y yo fuimos una" (Montañez, 2012, p. 34). ${ }^{12}$
En este cuento, la escritura se convierte en el espacio que permite bregar con la fealdad, gracias a que es la escritura la que le abre las puertas a Mena al plano de lo estético: "La escritura aminoró mi odio hacia todo lo no estético [...] La palabra me rescató de ese pozo; ahora la vejez en complot con la muerte me rescata de un segundo pozo infernal" (Montañez, 2012, p. 35).

Al final del relato, el lector se da cuenta, en parte por el cambio en la grafía del texto, que ha estado leyendo el diario de una Mena que ha llegado a la vejez. Mediante la negociación constante con lo feo y lo hermoso, y con el amor y el odio, Mena llega al punto en el cual se siente preparada para morir en relativa paz. Si las cicatrices causadas por el agua hirviendo forman un texto que se lee en el cuerpo de Mena y la excluye socialmente, su diario es un texto que nos permite leer otra narrativa de su vida. En esta narrativa, Mena no es victimizada sino que se convierte en una diva por haber superado la violencia física y emocional.

Un último ejemplo de la diva que brega con la violencia es el de Ana Flecha. Tras varios años de matrimonio, Juan, su marido, se vuelve un alcohólico que cada viernes -al llegar del barrompe la vajilla y los enseres domésticos. La trifulca se repite semana tras semana y Ana se da

$\overline{12}$ Las cursivas son del original. 
cuenta que la violencia hogareña no disminuirá. Ana decide tomar cartas en el asunto y compra una vajilla nueva para el próximo viernes. El texto indica que ese día "se levantó con la palabra 'basta' dándole vueltas por la cabeza" (Montañez, 2012, p. 55). Cuando su marido llega borracho, es ella la que destroza todas las piezas de la recién comprada vajilla. El marido la mira aterrado $\mathrm{y}$ le pide que no siga destruyendo las cosas. Al final, Juan logra ver en Ana el reflejo de sí mismo y cambia su carácter violento para que la familia vuelva a vivir en armonía.

\section{Conclusiones preliminares}

Estos personajes femeninos desafían las ideas generalizadas de lo que se entiende por una diva. Montañez se apropia del término y lo usa de una manera que le permite incorporar y exaltar subjetividades femeninas de la clase trabajadora, cuyos patrones de belleza no necesariamente encajan con los privilegiados dentro de una sociedad machista que ha convertido el cuerpo y su imagen en un signo de superioridad a medida que esté más hecho, más manufacturado. ${ }^{13}$ En otras palabras, "a medida que la mujer pueda y tenga más acceso para modificar y remodelar su cuerpo, para comprarse un cuerpo que después pueda intercambiar por aceptación social; a medida que una mujer contemporánea pueda tratar su cuerpo según su valor de cambio, es más mujer" (SantosFebres, 2005, pp. 54-55). Es por eso que hay cuerpos que están a las márgenes del mercado y suelen permanecer invisibles. Es decir, hay un sinnúmero de cuerpos que quedan fuera del centro, al no tener la salud ni el poder adquisitivo para adaptarse a los parámetros de femineidad favorecidos socialmente. A eso se le suma la relación entre pobreza y violencia que oprime a tantas mujeres en una sociedad machista. Aunque no propone soluciones fáciles ni todos los cuentos tienen finales felices, Montañez trae representaciones de estos asuntos y de estos cuerpos al centro, y expresa que la brega, que no perpetúa el papel de víctima ante la pobreza, la violencia y la discapacidad, tiene su toque de divinidad y es también digna de encomio.

\footnotetext{
13 Recordemos que entre los personajes de Las divas de mi barrio hay quienes son zambas, tienen las manos demasiado
} grandes, tienen llagas o cicatrices, tienen quemaduras, sufren de trastornos mentales y padecen de obesidad mórbida. 


\section{la palabra}

\section{Referencias}

Álvarez Curbelo, S. (2001). Un país del porvenir: el afán de modernidad en Puerto Rico (siglo XIX). San Juan: Ediciones Callejón.

Aronna, M. (1999). «Pueblos Enfermos»: the Discourse of Illness in the Turn-of-the-century Spanish and Latin American Essay. Chapel Hill: U.N.C. Department of Romance Languages.

Barradas, E. (1983). Palabras apalabradas: prólogo para una antología de cuentistas puertorriqueños de hoy. En: E. Barradas. Apalabramiento (pp.xiii-xxxi). Hanover: Ediciones del Norte.

Brau, S. (1956). Disquisiciones sociológicas y otros ensayos. Puerto Rico: Universidad de Puerto Rico.

Brown, K. (2010). Writing the Black Revolutionary Diva: Women's Subjectivity and the Decolonizing Text. Bloomington: Indiana University Press.

Clément, C. (1988). Opera, or, the Undoing of Women. Minneapolis: University of Minnesota.

Díaz Quiñones, A. (2000). El arte de bregar: Ensayos. San Juan, P.R.: Ediciones Callejón.

Ferré, R. (2000). Papeles de pandora. New York: Vintage.

Gelpí, J. (2005). Literatura y paternalismo en Puerto Rico. San Juan: Editorial de la Universidad de Puerto Rico.

Hind, E. (2010). Femmenism and the Mexican Woman Intellectual from Sor Juana to Poniatowska: Boob Lit. New York: Palgrave Macmillan.

Iriarte, M. (2015). Violencia y acceso a la justicia de las mujeres. San Juan: 80 grados. Recuperado el 5 de agosto de 2015, de http://www.80grados.net/violencia-y-acceso-a-la-justicia-de-las-mujeres/

Leonardi, S., y Pope, R. (1996). The Diva's Mouth: Body, Voice, Prima Donna Politics. New Brunswick, NJ: Rutgers University Press.

Montañez, C. (2012). Las divas de mi barrio. Bloomington: Palibrio.

Pedreira, A.S. (1942). Insularismo. San Juan: Biblioteca de Autores Puertorriqueños.

Sánchez, L.R. (1976). La guaracha del Macho Camacho. Buenos Aires: Ediciones de la Flor.

Santos-Febres, M. (2005). Sobre piel y papel. San Juan: Ediciones Callejon.

Smitherman, G. (2000). Black Talk: Words and Phrases from the Hood to the Amen Corner. Boston: Houghton Mifflin.

Zeno Gandía, M. (1955). Obras completas. Río Piedras: Ediciones del Instituto de Literatura Puertorriqueña. 